\title{
Article \\ On Consensus Index of Triplex Star-like Networks: A Graph Spectra Approach
}

\author{
Da Huang ${ }^{1, *}$, Jian Zhu ${ }^{1}$, Zhiyong $\mathrm{Yu}^{2}$ and Haijun Jiang ${ }^{2}$ \\ 1 Department of Mathematics and Physics, Xinjiang Institute of Engineering, Urumqi 830023, China; \\ zj17@xjie.edu.cn \\ 2 College of Mathematics and System Sciences, Xinjiang University, Urumqi 830046, China; \\ yzygsts@xju.edu.cn (Z.Y.); jianghai@xju.edu.cn (H.J.) \\ * Correspondence: hd@xjie.edu.cn
}

check for updates

Citation: Huang, D.; Zhu, J.; Yu, Z.; Jiang, H. On Consensus Index of Triplex Star-like Networks: A Graph Spectra Approach. Symmetry 2021, 13, 1248. https://doi.org/10.3390/ sym 13071248

Academic Editor: Lorentz Jäntschi

Received: 27 May 2021

Accepted: 9 July 2021

Published: 12 July 2021

Publisher's Note: MDPI stays neutral with regard to jurisdictional claims in published maps and institutional affiliations.

Copyright: (c) 2021 by the authors. Licensee MDPI, Basel, Switzerland. This article is an open access article distributed under the terms and conditions of the Creative Commons Attribution (CC BY) license (https:// creativecommons.org/licenses/by/ $4.0 /)$.

\begin{abstract}
In this article, the consensus-related performances of the triplex multi-agent systems with star-related structures, which can be measured by the algebraic connectivity and network coherence, have been studied by the characterization of Laplacian spectra. Some notions of graph operations are introduced to construct several triplex networks with star substructures. The methods of graph spectra are applied to derive the network coherence, and some asymptotic behaviors of the indices have been derived. It is found that the operations of adhering star topologies will make the first-order coherence increase a constant value under the triplex structures as parameters tend to infinity, and the second-order coherence have some equality relations as the node related parameters tend to infinity. Finally, the consensus related indices of the triplex systems with the same number of nodes but non-isomorphic graph structures have been compared and simulated to verify the results.
\end{abstract}

Keywords: consensus; coherence; Cartesian product; convergence speed; robustness; Laplacian spectrum

\section{Introduction}

Consensus is a class of distributed coordination problems of multi-agent systems, and the essence of the problem is that all agents are required to achieve a sort of common state under the effects of some control strategy. In the consensus model, the agents are designed to have communications among the nodes based on the graph structures of networks so that they can accomplish the assigned tasks together effectively.

As a significant interdisciplinary field, consensus problems have aroused wide interest among scholars and researchers, and there is significant potential value in many areas on consensus such as sensor networks [1], neural networks [2], and robotic systems [3]. Researchers have done many good studies on consensus [1-26] from various perspectives or using study factors including the dynamic order, communication ways(continuous or discontinuous), topology types (fixed or switching), control methods(adaptive control, intermittent control, impulsive control, etc.), and the consensus related index.

To solve consensus problems, communication relations can also be interpreted by the graph of the networked system, and consensus-related indices, such as convergence speed [4,9-11], and network coherence [13-20], can be characterized by the Laplacian eigenvalues of the graph. Synchronization problems, which share similar control strategies and have the same essence with consensus problems, are always connected with the graph structure [27-33] and studied from the perspective of algebraic graph theory. These facts inspire us to apply the methods of graph spectra in the study of conesensus problems in a deeper way. Among these enlightening works, [4] has showed that the algebraic connectivity $\lambda_{2}$ of an undirected (or directed) graph can characterize the convergence speed of consensus problems. In [11], the authors studied how the Laplacian eigenvalues determine the robustness and give derivations for $\lambda_{2}$ and the $H_{2}$ norms of several classic graphs. 
References [13-15] have studied the robustness of first and second order systems due to the $\mathrm{H}_{2}$ norm, and it has been proven that the network coherence can be characterized through all nonzero Laplacian eigenvalues. Reference [17] has studied the network coherence for a class of tree networks and found that the scalings of network coherence are smaller than some studied tree graphs. Reference [19] constructed the network with iterated line graphs of multisubdivision graph and obtained the scalings of the network coherence.

A network composed of interacting subnetworks of different (or similar) properties in structure and dynamics can be called a multilayer network. A multilayer network is a frontier research branch of network science, and its multilayered structure has many examples in real life, for example, the interactions between power grid and the Internet, friendship relations, or transportation and aviation networks. Multilayered networks have been studied from many perspectives, including multi-agent system-related problems [26,34], synchronization problems [30], epidemic propagation problems [35], etc. Multiplex networks are coupled multilayer networks where each layer is composed of the same copy of node set but possibly different graph structures, and the inter-layer links exist only between the counterpart nodes in different layers [36]. Considering the multilayer structures of multi-agent systems and the study of Laplacian-eigenvalue-related synchronizability problems $[28,29]$ with multiplex structures, it is natural to think that extending the consensus-index-related problem to multilayered graph structures is necessary and meaningful.

The star network is a type of classic computer network type. In fact, a star network can also be interpreted as a simplified model of the subnetworks of BA scale-free networks in the sense of statistics. There is in-depth research on star-related structures $[24,28]$ in many fields including synchronization, consensus problem, sensor networks, etc.

Inspired by this notion, this paper considers some three-layered networks with certain meaningful topologies that are constructed with graph operations; each layer contains star subgraphs, and the triplex networks with star subgraphs in this work are interpreted as adding communication links between the counterpart nodes for different layers of the networks. This paper makes further efforts to apply the theory of graph spectra for studying consensus indices in a deeper way.

This work mainly studies consensus indices of the networked systems with additive stochastic disturbances with an application of graph spectra methods. Specifically, the novelties of this paper are as follows:

1. Several novel layered star-like networks with different linking structures but the same number of nodes have been constructed by applying graph operations.

2. Graph spectra methods are applied to derive the Laplacian spectrums through the calculations of network coherence, and several new results on the asymptotic behavior have been acquired.

3. This article gives the notion that some nodes with low degree, for instance, the leaf nodes, might only have connections within the same layer other than connections with the counterpart nodes in different layers.

The paper is organized as follows. In Section 2, some graph operations are introduced, and the relations between the consensus indices and Laplacian spectrum are explained. In Section 3, the topological structure of the triplex systems are explained, and the main results are given. In Section 4, simulations are given and the comparison of performances for the four sorts of networks are made.

\section{Preliminaries}

\subsection{Graph Theory and Notations}

In this paper, all the graphs are considered undirected and connected. A star graph with $k$ leaves is denoted by $S_{k}$. $\mathfrak{E}_{k}$ denotes the empty graph with $\mathrm{k}$ vertices. Let $G$ be a undirected and connected graph with vertex set $V=\left\{v_{1}, v_{2}, \ldots, v_{N}\right\}$, and the edge set of $G$ is denoted by $\mathcal{E}=\{(i, j) \mid i, j=1,2, \ldots, N ; i \neq j\}$. The adjacency matrix of $G$, i.e., $A(G)$, is defined as $A(G)=\left[a_{i j}\right]_{N}$, where $a_{i j}$ is the weight of the edge $(i, j)$. All the edges 
in these networks are supposed to be 0 -1-weighted, that is, $a_{i j}=\left\{\begin{array}{ll}1, & (i, j) \in \mathcal{E} \text {; } \\ 0, & (i, j) \notin \mathcal{E} .\end{array}\right.$ The Laplacian matrix of $G$ is denoted by $L(G)$, and $L(G)=D(G)-A(G)$, where $D(G)$ is the diagonal degree matrix of $G$ defined by $D(G)=\operatorname{diag}\left(d_{1}, d_{2}, \ldots, d_{N}\right)$ with $d_{i}=\sum_{j \neq i} a_{i j}$.

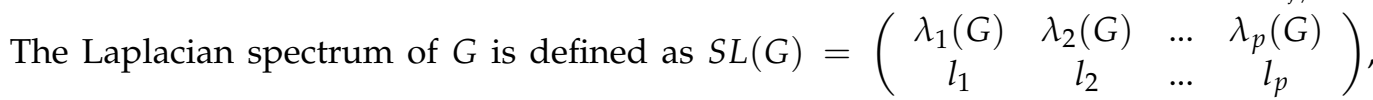
where $\lambda_{1}(G)<\lambda_{2}(G)<\ldots<\lambda_{p}(G)$ are the eigenvalues of $L(G)$ and $l_{1}, l_{2}, \ldots, l_{p}$ are the multiplicities of the eigenvalues. For any undirected graph, $\lambda_{1}=0$ is an eigenvalue of $L(G)$ associated with the eigenvector $\mathbf{1}=(1,1, \ldots, 1)$, and $L(G)$ is positive semi-definite and real symmetric.

To construct the triplex networks, the following graph operations are needed.

Definition $1\left([37,38]\right.$ (The corona of two graphs)). Let $G_{1}$ and $G_{2}$ be two graphs on disjoint sets of $n$ and $k$ vertices, respectively. The corona $G_{1} \circ G_{2}$ of $G_{1}$ and $G_{2}$ is defined as the graph obtained by taking one copy of $G_{1}$ and $n$ copies of $G_{2}$ and then joining the ith vertex of $G_{1}$ to every vertex in the ith copy of $G_{2},(i=1,2, \ldots, n)$.

Definition 2 ([39,40] (The Cartesian product of two graphs)). For two graphs $G_{1}=\left(V_{1}, E_{1}\right)$ and $G_{2}=\left(V_{2}, E_{2}\right)$, the Cartesian product graph $G=G_{1} \times G_{2}$ is the graph with vertex set $V_{1} \times V_{2}$; there is an edge from the vertex $\left(x_{1}, y_{1}\right)$ to the vertex $\left(x_{2}, y_{2}\right)$ if and only if either $x_{1}=x_{2}$ and $y_{1}, y_{2} \in E_{2}$ or $y_{1}=y_{2}$ and $x_{1}, x_{2} \in E_{1}$.

Definition 3 ([39] (The conjunction of two graphs)). The conjunction of simple graphs $G$ and $H$, written $G \nabla H$, is the graph obtained from the disjoint union of $G$ and $H$ by adding the edges $\{x y: x \in V(G), y \in V(H)\}$.

Lemma 1 ([37,38]). Let $G_{1}$ be any graph with $n_{1}$ vertices and $m_{1}$ edges, and let $G_{2}$ be any graph with $n_{2}$ vertices, $m_{2}$ edges. Suppose that $S L\left(G_{1}\right)=\left(\mu_{1}, \mu_{2}, \ldots, \mu_{n_{1}}\right)$ and $S L\left(G_{2}\right)=$ $\left(\delta_{1}, \delta_{2}, \ldots, \delta_{n_{2}}\right)$. Then the Laplacian spectrum of $G_{1} \circ G_{2}$ is given by

(i) Two multiplicity one eigenvalues $\frac{\left(\mu_{i}+n_{2}+1\right) \pm \sqrt{\left(\mu_{i}+n_{2}+1\right)^{2}-4 \mu_{i}}}{2} \in S L\left(G_{1} \circ G_{2}\right)$ for each eigenvalue $\mu_{i}\left(i=1,2, \ldots, n_{1}\right)$ of $S L\left(G_{1}\right)$;

(ii) $\delta_{j}+1 \in S L\left(G_{1} \circ G_{2}\right)$ with multiplicity $n_{1}$ for every eigenvalue $\delta_{j}\left(j=2, \ldots, n_{2}\right)$ of $S L\left(G_{2}\right)$.

Lemma 2 ([41]). If $G$ has $m$ vertices and $H$ has $n$ vertices, then the Laplacian eigenvalues of $G \times H$ are the $m n$ numbers: $v_{i}(G)+v_{j}(H)(i=1,2 \ldots, m ; j=1,2, \ldots, n)$.

Lemma 3 ([41]). If $G_{1}$ has $m$ vertices and $G_{2}$ has $n$ vertices, then the Laplacian eigenvalues of $G_{1} \nabla G_{2}$ are: $0, m+n, m+\lambda_{i}, n+\mu_{j}, i=2,3, \ldots, n ; j=2,3, \ldots, m$.

\subsection{Descriptions of Consensus Index with Laplacian Spectrum}

The main objective for this work is to investigate the robustness of the triplex systems when the dynamics have external disturbation and to accurately quantify the relations between the consensus indices and Laplacian eigenvalues. The robustness of the systems with noise can be described by the network coherence; in addition, the convergence speed, which can be characterized by $\lambda_{2}$ (algebraic connectivity), is discussed.

(i) First order case.

In this case, the networked system with additive noise can be written as

$$
\dot{x}(t)=-L(G) x(t)+\varsigma(t)
$$

where $x(t) \in R^{N}$, and $\varsigma(t) \in R^{N}$ is a vector of delta-correalted Gaussian noise, and $L(G)$ is the Laplacian matrix of $G$. 
Definition 4 ([13-15]). The first-order network coherence is defined as the mean steady-state variance of the deviation from the average of all node values:

$$
H_{f}:=\lim _{t \rightarrow \infty} \frac{1}{N} \sum_{i=1}^{N} \operatorname{Var}\left\{x_{i}(t)-\frac{1}{N} \sum_{j=1}^{N} x_{j}(t)\right\}
$$

It has been proven that $([12,13,15])$ the first-order coherence $H_{f}$ can be completely characterized by the Laplacian eigenvalues. Let the Laplacian eigenvalues be described by $0=\lambda_{1}<\lambda_{2} \leq \ldots \leq$ $\lambda_{N}$; then, the first-order coherence can be determined by

$$
H_{f}=\frac{1}{2 N} \sum_{i=2}^{N} \frac{1}{\lambda_{i}}
$$

\section{(i) Second order case.}

In the second order network, such as vehicle formation problem, there are $N$ vehicles. The states of a nodes have a position vector $x(t) \in R^{N}$ and a velocity vector $v(t) \in R^{N}$. The network can be written as

$$
\left[\begin{array}{c}
x \dot{(t)} \\
v(t)
\end{array}\right]=\left[\begin{array}{cc}
0 & I \\
-L(G) & -L(G)
\end{array}\right]\left[\begin{array}{l}
x(t) \\
v(t)
\end{array}\right]+\left[\begin{array}{l}
0 \\
I
\end{array}\right] \zeta(t)
$$

where $\varsigma(t)$ is a $2 N$-vector of zero mean white noise processes and I is the identity matrix.

Definition 5. The second-order network coherence is defined in terms of $x(t)$, and it describes the deviation from the average of of the first state variable of one nodes, and the definition has an identical form with the first-order case. i.e.,

The second-order coherence can also be characterized by Laplacian eigenvalues [15],

$$
H_{s}=\frac{1}{2 N} \sum_{i=2}^{N} \frac{1}{\lambda_{i}^{2}}
$$

The notion of network coherence implies the ability of maintaining its convergence trend under the effect of stochastic disturbances. The characterization of this consensus index has some similarity with the Kirhoff index [42,43].

\section{Model Description and Main Results}

As mentioned in Section 1, the layered star-like network in this paper is a kind of network in which all nodes have identical form of dynamics, and it has the topology composed by linking the center nodes among basic star topologies. The graphs in this article are undirected and connected. The following subsections are given to define the four types of networks and calculate the consensus indices.

\subsection{The Consensus Indices for Network Topology $G(\mathbb{A})$}

The topology in the same layer can be viewed as two star graph structures sharing the same leaf nodes, and for which there are communication links between the center nodes. Between the pair of nodes with corresponding position of different layers of the triplex network, they are connected with an edge of the path $P_{3}$.

It is obvious that the network topology can be characterized by $\left(P_{2} \nabla \mathfrak{E}_{n}\right) \times P_{3}$, and the network is denoted by $\mathbb{A}$ (see Figure 1). 


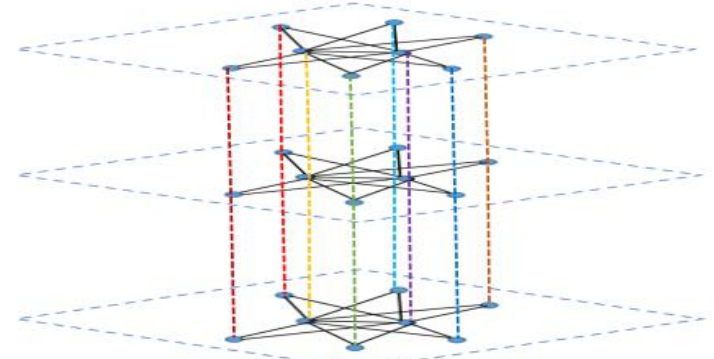

Figure 1. An example of the topology $G(\mathbb{A}), n=6$.

Since $S L\left(P_{2}\right)=\left(\begin{array}{ll}0 & 2 \\ 1 & 1\end{array}\right), S L\left(\mathfrak{E}_{n}\right)=\left(\begin{array}{l}0 \\ n\end{array}\right)$, and since $S L\left(P_{3}\right)=\left(\begin{array}{ccc}0 & 1 & 3 \\ 1 & 1 & 1\end{array}\right)$, then, by Lemmas 2 and 3, one has

$$
S L[G(\mathbb{A})]=\left(\begin{array}{ccccccccc}
0 & 3+n & 1 & 3 & 3 & 5+n & 5 & 2+n & 2 \\
1 & 2 & 1 & n-1 & 1 & 2 & n-1 & 2 & n-1
\end{array}\right)
$$

then the first-order coherence of network $\mathbb{A}$ can be derived as:

$$
\begin{aligned}
H^{(1)}(\mathbb{A})= & \frac{1}{2 N} \sum_{i=2}^{N} \frac{1}{\lambda_{i}}=\frac{1}{2(3 n+6)}\left[1+\frac{1}{3+n}+\frac{1}{4}+\frac{1}{3}(n-1)+\frac{1}{3}+\frac{1}{5+n}\right. \\
& \left.+\frac{1}{6}+\frac{1}{5}(n-1)+\frac{1}{2+n}+\frac{1}{3}+\frac{1}{2}(n-1)\right] \\
= & \frac{1}{6 n+12}\left(\frac{25}{12}+\frac{31}{30}(n-1)+\frac{1}{3+n}+\frac{1}{5+n}+\frac{1}{2+n}\right) \\
\rightarrow & \frac{31}{180} \approx 0.172(n \rightarrow \infty)
\end{aligned}
$$

The second-order coherence of $\mathbb{A}$ can be calculated as:

$$
\begin{aligned}
H^{(2)}(\mathbb{A})= & \frac{1}{2 N} \sum_{i=2}^{N} \frac{1}{\lambda_{i}^{2}}=\frac{1}{6 n+12}\left(1+\frac{1}{(3+n)^{2}}+\frac{1}{16}+\frac{n-1}{9}+\frac{1}{9}+\frac{1}{(5+n)^{2}}+\frac{1}{36}\right. \\
& \left.+\frac{(n-1)}{25}+\frac{1}{(2+n)^{2}}+\frac{1}{9}+\frac{(n-1)}{4}\right) \rightarrow \frac{361}{5400} \approx 0.067(n \rightarrow \infty) .
\end{aligned}
$$

\subsection{The Consensus Indices for Network Topology $G(\mathbb{B})$}

The topology in the same layer can be seen as a wheel graph, a wheel graph structure can be interpreted as adding edges between the leaf vertices of the star graph, and the original leaf nodes form into a circle. One can see that in this construction design, the network $\mathbb{A}$ and $\mathbb{B}$ has the same number of nodes in one layer, but the ling structure is different. The network topology $G(\mathbb{B})$ can be described as $\left(\mathfrak{E}_{1} \nabla C_{n+1}\right) \times P_{3}$ (see Figure 2), where $C_{n+1}$ denotes the circle with $n+1$ nodes.

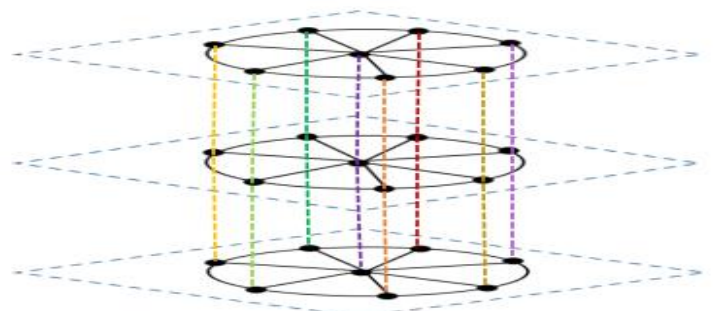

Figure 2. An example of $G(\mathbb{B}):\left(\mathfrak{E}_{1} \nabla C_{n+1}\right) \times P_{3}, n=6$. 


$$
\begin{aligned}
& \text { Since } S L\left(C_{n+1}\right)=\left(0,4 \sin ^{2}\left(\frac{k \pi}{n+1}\right)\right), k=1,2, \ldots, n, \text { one has } \\
& S L\left(\mathfrak{E}_{1} \nabla C_{n+1}\right)=\left(\begin{array}{ccc}
0 & n+2 & 1+4 \sin ^{2}\left(\frac{k \pi}{n+1}\right) \\
1 & 1 & n
\end{array}\right)
\end{aligned}
$$

and since $S L\left(P_{3}\right)=\left(\begin{array}{lll}0 & 1 & 3 \\ 1 & 1 & 1\end{array}\right)$, by Lemmas $2-3$, the Laplacian spectrum of $G(\mathbb{B})$ has the following form:

$$
\begin{aligned}
& S L(G(\mathbb{B}))= \\
& \left(\begin{array}{ccccccccc}
0 & 1 & 3+n & 2+4 \sin ^{2}\left(\frac{k \pi}{n+1}\right) & 3 & 5+n & 4+4 \sin ^{2}\left(\frac{k \pi}{n+1}\right) & n+2 & 1+4 \sin ^{2}\left(\frac{k \pi}{n+1}\right) \\
1 & 1 & 1 & n & 1 & 1 & n & 1 & n
\end{array}\right)
\end{aligned}
$$

From the Laplacian spectrum, one can see that $\lambda_{2}=1$. Thus, it has the same convergence speed with $\mathbb{A}$.

Therefore, the first-order coherence of network $\mathbb{B}$ can be derived as:

$$
\begin{aligned}
H^{(1)}(\mathbb{B})= & \frac{1}{2 N} \sum_{i=2}^{N} \frac{1}{\lambda_{i}}=\frac{1}{6 n+12} \cdot\left(1+\frac{1}{3+n}+\sum_{k=1}^{n} \frac{1}{2+4 \sin ^{2}\left(\frac{k \pi}{n+1}\right)}+\frac{1}{3}+\frac{1}{5+n}\right. \\
& \left.+\sum_{k=1}^{n} \frac{1}{4+4 \sin ^{2}\left(\frac{k \pi}{n+1}\right)}+\frac{1}{n+2}+\sum_{k=1}^{n} \frac{1}{1+4 \sin ^{2}\left(\frac{k \pi}{n+1}\right)}\right),
\end{aligned}
$$

then

$$
\begin{aligned}
\lim _{n \rightarrow \infty} H^{(1)}(\mathbb{B}) & =\frac{1}{12} \int_{0}^{1} \frac{1}{1+2 \sin ^{2} \pi x} d x+\frac{1}{24} \int_{0}^{1} \frac{1}{1+\sin ^{2}(\pi x)} d x+\frac{1}{6} \int_{0}^{1} \frac{1}{1+4 \sin ^{2} \pi x} d x \\
& =\frac{\sqrt{3}}{36}+\frac{\sqrt{2}}{48}+\frac{\sqrt{5}}{30} \approx 0.152 .
\end{aligned}
$$

The second-order coherence of $\mathbb{B}$ can be derived as:

$$
\begin{aligned}
H^{(2)}(\mathbb{B})= & \frac{1}{2 N} \sum_{i=2}^{N} \frac{1}{\lambda_{i}^{2}}=\frac{1}{6 n+12}\left(1+\frac{1}{(3+n)^{2}}+\sum_{k=1}^{n} \frac{1}{\left[2+4 \sin ^{2}\left(\frac{k \pi}{n+1}\right)\right]^{2}}+\frac{1}{9}\right. \\
& \left.+\frac{1}{(5+n)^{2}}+\sum_{k=1}^{n} \frac{1}{\left[4+4 \sin ^{2}\left(\frac{k \pi}{n+1}\right)\right]^{2}}+\frac{1}{(n+2)^{2}}+\sum_{k=1}^{n} \frac{1}{\left[1+4 \sin ^{2}\left(\frac{k \pi}{n+1}\right)\right]^{2}}\right)
\end{aligned}
$$

then

$$
\begin{aligned}
\lim _{n \rightarrow \infty} H^{(2)}(\mathbb{B})= & \frac{1}{24} \int_{0}^{1} \frac{1}{\left[1+2 \sin ^{2}(\pi x)\right]^{2}} d x+\frac{1}{96} \int_{0}^{1} \frac{1}{\left[1+\sin ^{2}(\pi x)\right]^{2}} d x+ \\
& \frac{1}{6} \int_{0}^{1} \frac{1}{\left[1+4 \sin ^{2}(\pi x)\right]^{2}} d x \\
= & \frac{\sqrt{3}}{108}+\frac{\sqrt{2}}{64}+\frac{\sqrt{5}}{50} \approx 0.067
\end{aligned}
$$

\subsection{The Consensus Indices for Network Topology $G(\overline{\mathbb{A}})$}

In real applications, there may exist nodes with low degree that connect others only within the same layer but do not have connection with other layers. It should be noted that in the case of Sections 3.3 and 3.4, the leaf node in one layer is designed to be disconnected from other layers.

In this subsection, a sort of triplex star-like graph with symmetric structure based on $\mathbb{A}$ is considered. 
As shown in Figure 3, let each node in $\overline{\mathbb{A}}$ be the center nodes sticking to a star structure with $k$ leaf nodes; then $G(\overline{\mathbb{A}})=G(\mathbb{A}) \circ \mathfrak{E}_{k}, k \geq 3$, i.e., $\left[\left(P_{2} \nabla \mathfrak{E}_{n}\right) \times P_{3}\right] \circ \mathfrak{E}_{k}$. The leaf nodes are designed to disconnect with other layers.

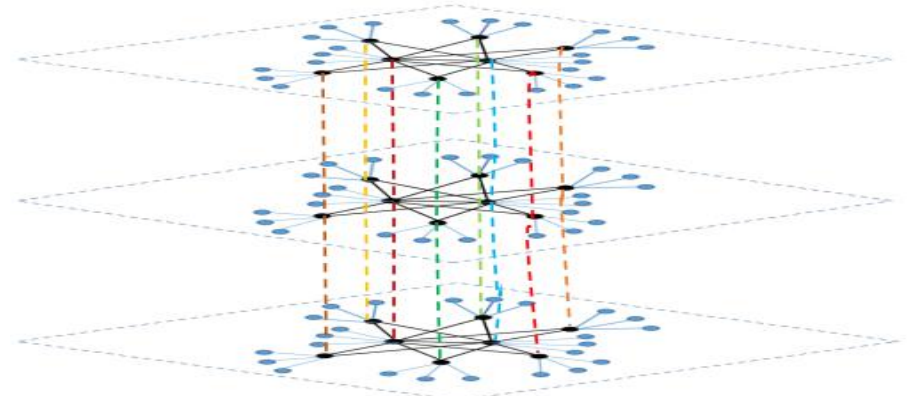

Figure 3. An example of $G(\overline{\mathbb{A}}), n=6, k=3$.

By Lemma 1, the Laplacian spectrum of $G(\overline{\mathbb{A}})$ has the following description:

(1) $0 \in S L(G(\overline{\mathbb{A}}))$ and $k+1 \in S L(G(\overline{\mathbb{A}}))$ with multiplicity 1 ;

(2) $\frac{2+k \pm \sqrt{(2+k)^{2}-4}}{2} \in S L[G(\overline{\mathbb{A}})]$ with multiplicity 1;

(3) $\frac{4+k \pm \sqrt{(4+k)^{2}-12}}{2} \in S L[G(\overline{\mathbb{A}})]$ with multiplicity $n$;

(4) $\frac{4+n+k \pm \sqrt{(4+n+k)^{2}-4(3+n)}}{2} \in S L[G(\overline{\mathbb{A}})]$ with multiplicity 2;

(5) $\frac{6+n+k \pm \sqrt{(6+n+k)^{2}-4(5+n)}}{2} \in S L[G(\overline{\mathbb{A}})]$ with multiplicity 2;

(6) $\frac{6+k \pm \sqrt{(6+k)^{2}-20}}{2} \in S L[G(\overline{\mathbb{A}})]$ with multiplicity $(n-1)$;

(7) $\frac{3+n+k \pm \sqrt{(3+n+k)^{2}-4(2+n)}}{2} \in S L[G(\overline{\mathbb{A}})]$ with multiplicity 2;

(8) $\frac{3+k \pm \sqrt{(3+k)^{2}-8}}{2} \in S L[G(\overline{\mathbb{A}})]$ with multiplicity $(n-1)$.

(9) $1 \in S L[G(\overline{\mathbb{A}})]$ with multiplicity $3(n+2)(k-1)$.

From the Laplacian spectrum, one can see that $\lambda_{2}=\frac{2+k-\sqrt{(2+k)^{2}-4}}{2}$ for $G(\overline{\mathbb{A}})$.

Therefore, the first-order coherence of $\overline{\mathbb{A}}$ has the form:

$$
\begin{aligned}
H^{(1)}(\overline{\mathbb{A}})= & \frac{1}{2 N} \sum_{i=2}^{N} \frac{1}{\lambda_{i}}=\frac{1}{2(3 n+6)(k+1)}\left(\frac{1}{k+1}+(2+k)+\frac{(4+k)(n-1)}{3}\right. \\
& +\frac{2(4+n+k)}{3+n}+\frac{4+k}{3}+\frac{2(6+n+k)}{5+n}+\frac{(6+k)(n-1)}{5}+\frac{2(3+n+k)}{2+n} \\
& \left.+\frac{(3+k)(n-1)}{2}+3(n+2)(k-1)\right) .
\end{aligned}
$$

Therefore,

$$
\lim _{n, k \rightarrow \infty} H^{(1)}(\overline{\mathbb{A}})=\frac{121}{180} \approx 0.672
$$

Hence, one can see that adding the star topology does not change the first-order coherence of $\mathbb{A}$.

The second-order coherence of network $\overline{\mathbb{A}}$ can be calculated as: 


$$
\begin{aligned}
H^{(2)}(\overline{\mathbb{A}})= & \frac{1}{2 N} \sum_{i=2}^{N} \frac{1}{\lambda_{i}^{2}}=\frac{1}{2(3 n+6)(k+1)}\left(\frac{1}{(k+1)^{2}}+(2+k)^{2}-2\right. \\
& +(n) \frac{(4+k)^{2}-6}{9}+2 \frac{(4+n+k)^{2}-2(3+n)}{(3+n)^{2}}+2 \frac{(6+n+k)^{2}-2(5+n)}{(5+n)^{2}} \\
& +(n-1) \frac{(6+k)^{2}-10}{25}+2 \frac{(3+n+k)^{2}-2(2+n)}{(2+n)^{2}}+(n-1) \frac{(3+k)^{2}-4}{4} \\
& +3(n+2)(k-1)),
\end{aligned}
$$

Let $n, k \rightarrow \infty$; the asymptotic behaviour for the second-order coherence can be described as

$$
\lim _{n, k \rightarrow \infty} \frac{H^{(2)}(\overline{\mathbb{A}})}{k}=\frac{5}{24}+\frac{1}{150} \approx 0.215
$$

\subsection{The Consensus Indices for Network Topology $G(\overline{\mathbb{B}})$}

As shown in Figure 4, let each node in $\overline{\mathbb{B}}$ be the center nodes stick to a star structure with $k$ leaf nodes; then, $G(\bar{B})=G(\mathbb{B}) \circ \mathfrak{E}_{p}, p \geq 3$. The leaf nodes of this vertex's degree, equal to 1 , are designed to not have access to link to other layers. The number of basic center nodes (black ones in Figure 4) in one layer is same with $G(\overline{\mathbb{A}})$; however, the linking structure is different.

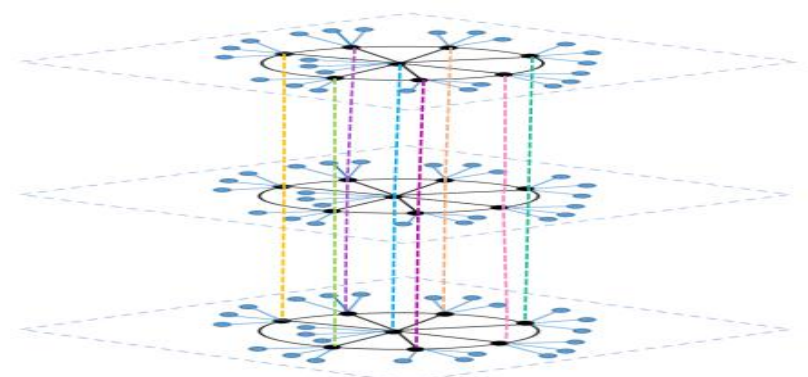

Figure 4. An example of $G(\overline{\mathbb{B}}):\left[\left(\mathfrak{E}_{1} \nabla C_{n+1}\right) \times P_{3}\right] \circ E_{p}, n=6, p=3$.

By Lemmas $1-3$, the Laplacian spectrum of $G(\bar{B})$ can be characterized as:

(1) $p+1,0 \in S L[G(\overline{\mathbb{B}})]$ with multiplicity 1 ;

(2) $\frac{2+p \pm \sqrt{(2+p)^{2}-4}}{2} \in S L[G(\overline{\mathbb{B}})]$ with multiplicity 1;

(3) $\frac{4+n+p \pm \sqrt{(4+n+p)^{2}-4(3+n)}}{2} \in S L[G(\overline{\mathbb{B}})]$ with multiplicity 1 ;

(4) $\frac{\left(3+4 \sin ^{2}\left(\frac{k \pi}{n+1}\right)+p\right) \pm \sqrt{\left(3+4 \sin ^{2}\left(\frac{k \pi}{n+1}\right)+p\right)^{2}-4\left(2+4 \sin ^{2}\left(\frac{k \pi}{n+1}\right)\right)}}{2} \in S L[G(\overline{\mathbb{B}})]$ with multiplicity 1,

$k=1,2, \ldots, n$

(5) $\frac{4+p \pm \sqrt{(4+p)^{2}-12}}{2} \in S L[G(\overline{\mathbb{B}})]$ with multiplicity 1 ;

(6) $\frac{6+n+p \pm \sqrt{(6+n+p)^{2}-4(5+n)}}{2} \in S L[G(\overline{\mathbb{B}})]$ with multiplicity 1 ;

(7) $\frac{\left(5+4 \sin ^{2}\left(\frac{k \pi}{n+1}\right)+p\right) \pm \sqrt{\left(5+4 \sin ^{2}\left(\frac{k \pi}{n+1}\right)+p\right)^{2}-4\left(4+4 \sin ^{2}\left(\frac{k \pi}{n+1}\right)\right)}}{2} \in S L[G(\overline{\mathbb{B}})]$ with multiplicity 1, $k=1,2, \ldots, n$;

(8) $\frac{3+n+p \pm \sqrt{(3+n+p)^{2}-4(2+n)}}{2} \in S L[G(\overline{\mathbb{B}})]$ with multiplicity 1;

(9) $\frac{\left(2+4 \sin ^{2}\left(\frac{k \pi}{n+1}\right)+p\right) \pm \sqrt{\left(2+4 \sin ^{2}\left(\frac{k \pi}{n+1}\right)+p\right)^{2}-4\left(1+4 \sin ^{2}\left(\frac{k \pi}{n+1}\right)\right)}}{2} \in S L[G(\overline{\mathbb{B}})]$ with multiplicity 1, $k=1,2, \ldots, n$.

(10) $1 \in S L[G(\overline{\mathbb{B}})]$ with multiplicity $3(n+2)(p-1)$.

Therefore, the convergence speed can be characterized as $\lambda_{2}=\frac{2+p-\sqrt{(2+p)^{2}-4}}{2}$. 
The first-order coherence of network $\overline{\mathbb{B}}$ can be calculated as:

$$
\begin{aligned}
H^{(1)}(\overline{\mathbb{B}}) & =\frac{1}{2 N} \sum_{i=2}^{N} \frac{1}{\lambda_{i}}=\frac{1}{6(n+2)(1+p)}\left(\frac{1}{p+1}+\frac{1}{p+2}+\frac{4+n+p}{3+n}\right. \\
& +\sum_{k=1}^{n}\left(1+\frac{1+p}{2+4 \sin ^{2}\left(\frac{k \pi}{n+1}\right)}\right)+\frac{4+p}{3}+\frac{6+n+p}{5+n}+\sum_{k=1}^{n}\left(1+\frac{1+p}{4+4 \sin ^{2}\left(\frac{k \pi}{n+1}\right)}\right) \\
& \left.+\frac{3+n+p}{2+n}+\sum_{k=1}^{n}\left(1+\frac{1+p}{1+4 \sin ^{2}\left(\frac{k \pi}{n+1}\right)}\right)+3(n+2)(p-1)\right)
\end{aligned}
$$

then

$$
\begin{aligned}
\lim _{n, p \rightarrow \infty} H^{(1)}(\overline{\mathbb{B}}) & =\frac{1}{12} \int_{0}^{1} \frac{1}{1+2 \sin ^{2}(\pi x)} d x+\frac{1}{24} \int_{0}^{1} \frac{1}{1+\sin ^{2}(\pi x)} d x \\
& +\frac{1}{6} \int_{0}^{1} \frac{1}{1+4 \sin ^{2}(\pi x)} d x+\frac{1}{2} \\
& =\frac{\sqrt{3}}{36}+\frac{\sqrt{2}}{48}+\frac{\sqrt{5}}{30}+\frac{1}{2} \approx 0.652
\end{aligned}
$$

Remark 1. Compared with the network $\mathbb{B}$, one can see that if the layered network has had star topologies added to each node, then the first-order coherence will increase at a constant value as $n, p \rightarrow \infty$; that is, $H^{(1)}(\overline{\mathbb{B}})=H^{(1)}(\mathbb{B})+0.5(n, p \rightarrow \infty)$, and the same relationship applies to $\mathbb{A}$ and $\overline{\mathbb{A}}$.

The second-order coherence of $\overline{\mathbb{B}}$ can be calculated as:

$$
\begin{aligned}
H^{(2)}(\overline{\mathbb{B}})= & \frac{1}{2 N} \sum_{i=2}^{N} \frac{1}{\lambda_{i}^{2}} \\
= & \frac{1}{6(n+2)(1+p)}\left(\sum _ { k = 1 } ^ { n } \left(\frac{p^{2}+2 p\left(2+4 \sin ^{2}\left(\frac{k \pi}{n+1}\right)\right)+\left(2+4 \sin ^{2}\left(\frac{k \pi}{n+1}\right)\right)^{2}}{\left(1+4 \sin ^{2}\left(\frac{k \pi}{n+1}\right)\right)^{2}}\right.\right. \\
& \left.-\frac{2}{1+4 \sin ^{2}\left(\frac{k \pi}{n+1}\right)}\right)+\sum_{k=1}^{n}\left(\frac{p^{2}+2 p\left(3+4 \sin ^{2}\left(\frac{k \pi}{n+1}\right)\right)+\left(3+4 \sin ^{2}\left(\frac{k \pi}{n+1}\right)\right)^{2}}{\left(2+4 \sin ^{2}\left(\frac{k \pi}{n+1}\right)\right)^{2}}\right. \\
& \left.-\frac{1}{1+2 \sin ^{2}\left(\frac{k \pi}{n+1}\right)}\right)+\sum_{k=1}^{n}\left(\frac{p^{2}+2 p\left(5+4 \sin ^{2}\left(\frac{k \pi}{n+1}\right)\right)+\left(5+4 \sin ^{2}\left(\frac{k \pi}{n+1}\right)\right)^{2}}{\left(4+4 \sin ^{2}\left(\frac{k \pi}{n+1}\right)\right)^{2}}\right. \\
& \left.-\frac{1}{2\left(1+\sin ^{2}\left(\frac{k \pi}{n+1}\right)\right)}\right)+\frac{1}{(p+1)^{2}}+(2+p)^{2}-2+\left(1+\frac{1+p}{3+n}\right)^{2}-\frac{2}{3+n} \\
& \left.+\left(1+\frac{1+p}{5+n}\right)^{2}-\frac{2}{5+n}+\left(1+\frac{1+p}{2+n}\right)^{2}-\frac{2}{2+n}+\frac{(4+p)^{2}-6}{9}\right)
\end{aligned}
$$

Therefore, the symptotic behavior of $H^{(2)}(\overline{\mathbb{B}})$ can be described as:

$$
\begin{aligned}
\lim _{n, p \rightarrow \infty} \frac{H^{(2)}(\overline{\mathbb{B}})}{p}= & \frac{1}{6} \int_{0}^{1} \frac{1}{\left(1+4 \sin ^{2}(\pi x)\right)^{2}} d x+\frac{1}{24} \int_{0}^{1} \frac{1}{\left(1+2 \sin ^{2}(\pi x)\right)^{2}} d x \\
& +\frac{1}{96} \int_{0}^{1} \frac{1}{\left(1+\sin ^{2}(\pi x)\right)^{2}} d x \\
= & \frac{\sqrt{5}}{50}+\frac{\sqrt{3}}{108}+\frac{\sqrt{2}}{256} \approx 0.067
\end{aligned}
$$




\section{Simulation and Comparison}

It should be emphasized that the numbers of nodes in each layer of $\mathbb{A}$ and $\mathbb{B}$ are the same, i.e., $|V(G(\mathbb{A}))|=|V(G(\mathbb{B}))|=n+2$, and the equality relation applies to $\overline{\mathbb{A}}$ and $\overline{\mathbb{B}}$ if the numbers of leaf nodes are also the same. The comparisons of performances for the triplex networks are made from two perspectives: vertically, $\mathbb{A}$ and $\overline{\mathbb{A}}, \mathbb{B}$ and $\overline{\mathbb{B}}$; horizontally, $\mathbb{A}$ and $\mathbb{B}, \overline{\mathbb{A}}$ and $\overline{\mathbb{B}}$. By the derivation of Section 3 , since $f(x)=\frac{x+2-\sqrt{(x+2)^{2}-4}}{2}$ is monotonically decreasing, the maximum convergence speed for $G(\overline{\mathbb{A}})$ and $G(\overline{\mathbb{B}})$ can be characterized by $\lambda_{2 \max }=\frac{5-\sqrt{21}}{2}<1$ and $\lambda_{2} \rightarrow 0$ as $p \rightarrow \infty$. The change in $\lambda_{2}$ is shown in Figure 5. Therefore, the network $\mathbb{A}$ and $\mathbb{B}$ reach consensus much slower than $\overline{\mathbb{A}}$ and $\overline{\mathbb{B}}$, respectively. From the previous discussion, one can see that the first-order coherence of $\overline{\mathbb{A}}$ is 0.5 greater than that of $\mathbb{A}$, and the relationship applies to $\mathbb{B}$ and $\mathbb{B}$, which means the robustness will increase at a fixed value when the operation of adding star topologies is made. As for the relation of second-order coherence for $\mathbb{B}$ and $\overline{\mathbb{B}}$, we have $\lim _{n, p \rightarrow \infty} H^{(2)}(\overline{\mathbb{B}})=p \lim _{n \rightarrow \infty} H^{(2)}(\mathbb{B})$.

From the calculation results and the simulations, one can see that the first-order coherence of $\mathbb{A}$ and $\mathbb{B}$ has the relation $H^{(1)}(\mathbb{A})>H^{(1)}(\mathbb{B})$ as $n \rightarrow \infty$, and for $\overline{\mathbb{A}}$ and $\overline{\mathbb{B}}$, we have $H^{(1)}(\overline{\mathbb{A}})>H^{(1)}(\overline{\mathbb{B}})$ as $n, k \rightarrow \infty$.

In the second-order case, $H^{(2)}(\mathbb{A}) \approx H^{(2)}(\mathbb{B})$ as $n, p \rightarrow \infty$. The value of $H^{(2)}(\overline{\mathbb{A}})$ mainly depends on the number of leaf nodes $k$ when $n, k$ are large enough.

$H^{(2)}(\overline{\mathbb{A}})$ is much larger than $H^{(2)}(\mathbb{A})$ when $n$ is large enough, which means the second order robustness of $\mathbb{A}$ is not as good as that of $\mathbb{A}$.

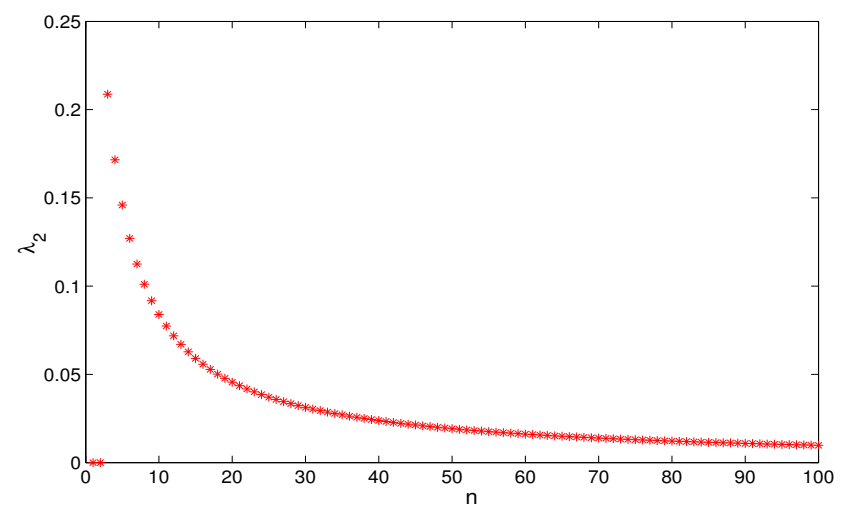

Figure 5. The change of $\lambda_{2}$ for $G(\overline{\mathbb{A}})$ and $G(\overline{\mathbb{B}})$.

The variances of the network coherence for the four networks are shown in Figures 6-13. It can be seen that the simulations verify the calculation results well.

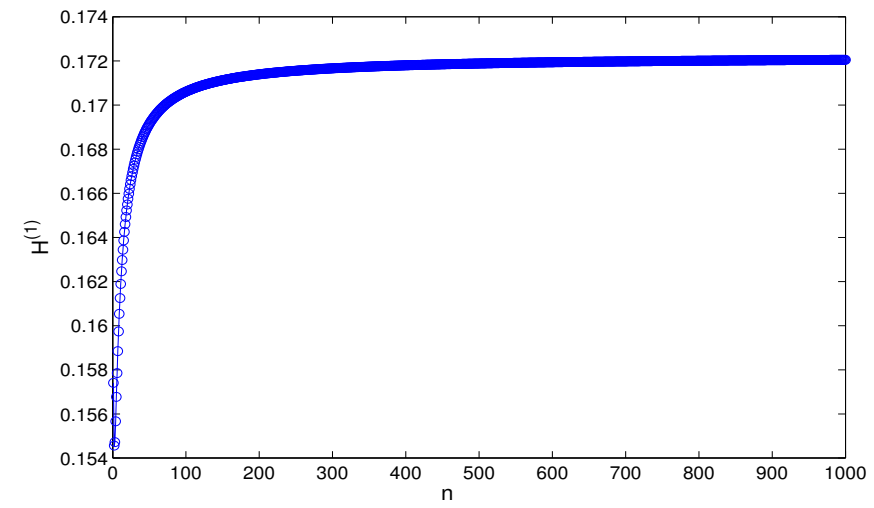

Figure 6. The change of $H^{(1)}$ for $\mathbb{A}$. 


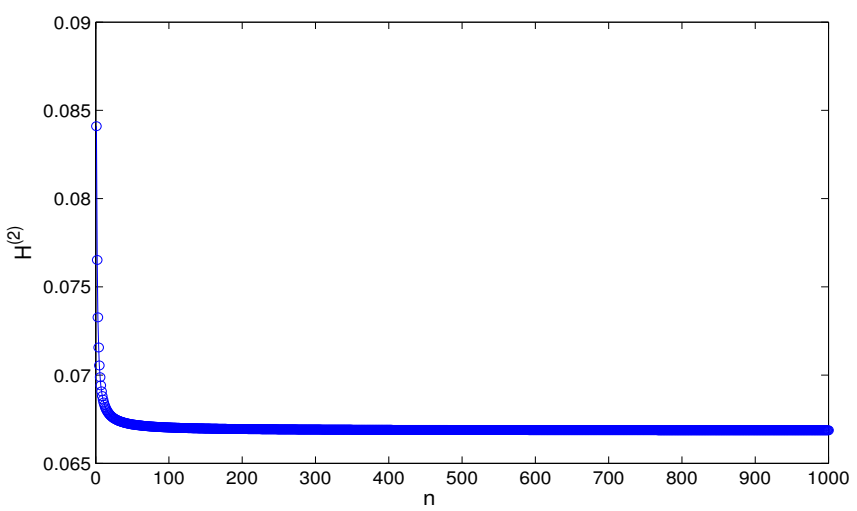

Figure 7. The change of $H^{(2)}$ for $\mathbb{A}$.

The variance of the network coherence for $\mathbb{B}$ is shown in Figures 8 and 9.

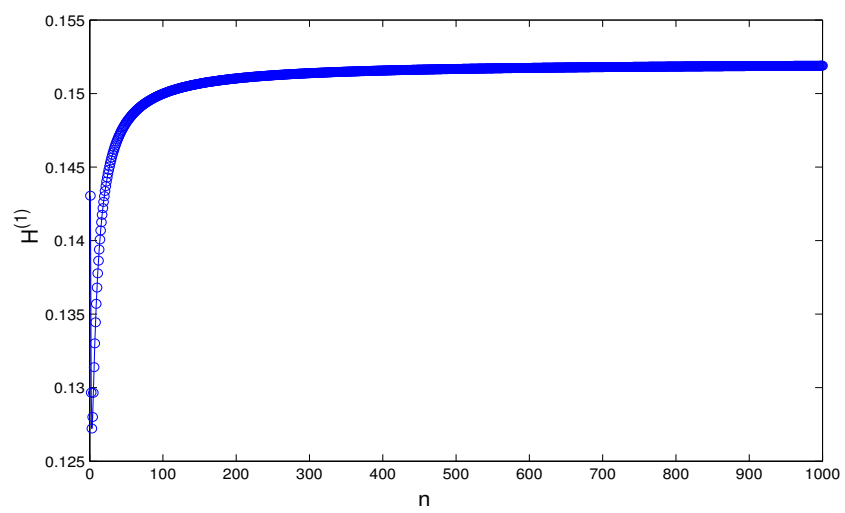

Figure 8. The change of $H^{(1)}$ for $\mathbb{B}$.

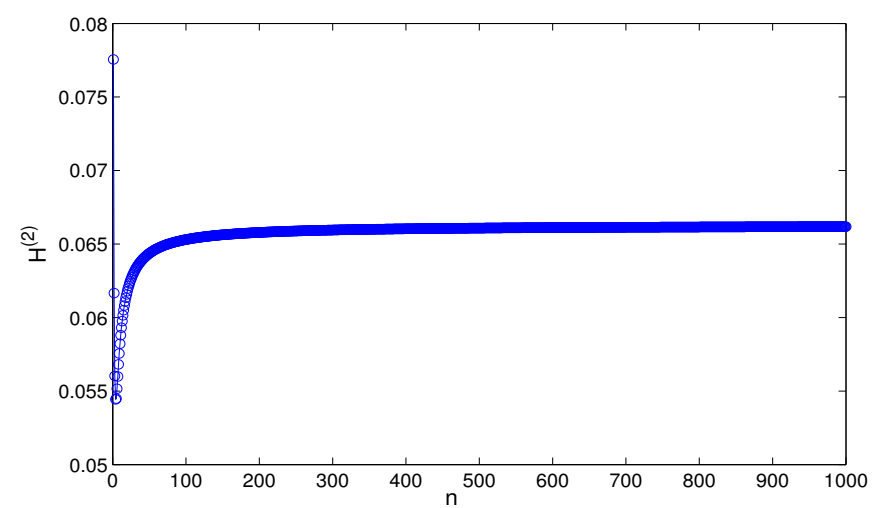

Figure 9. The change of $H^{(2)}$ for $\mathbb{B}$.

The variance of the network coherence for $\overline{\mathbb{A}}$ and $\overline{\mathbb{B}}$ is shown in Figures 10-13. 


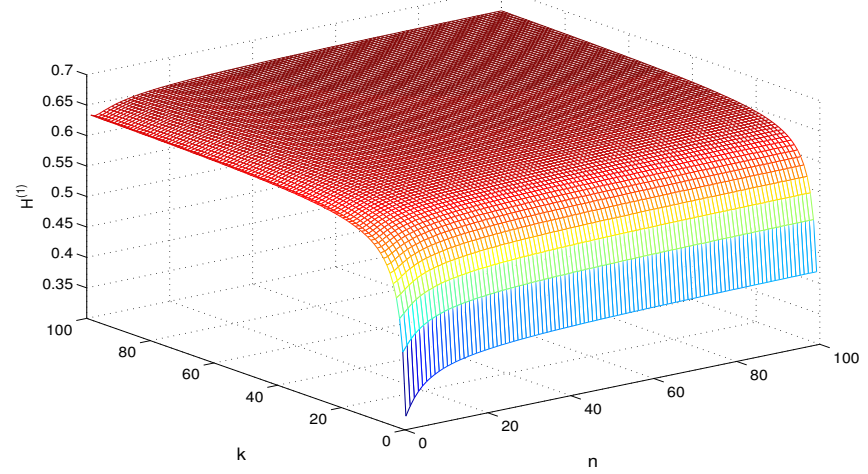

Figure 10. The change in $H^{(1)}$ for $\overline{\mathbb{A}}$.

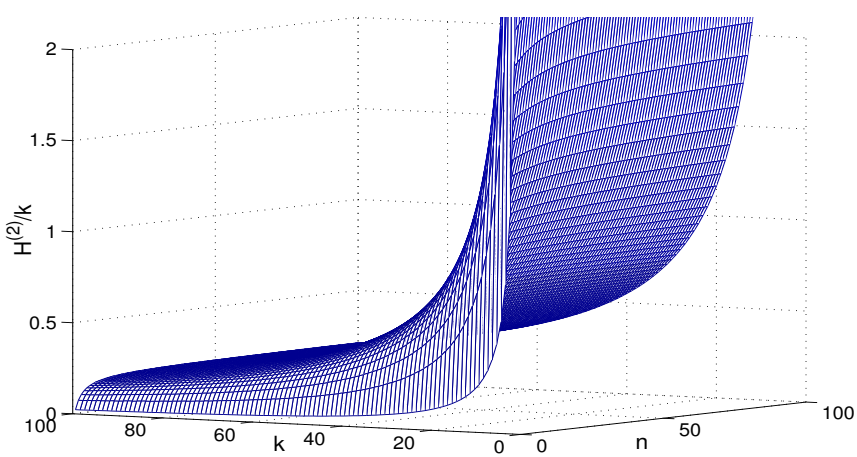

Figure 11. The change in $\frac{H^{(2)}}{k}$ for $\overline{\mathbb{A}}$.

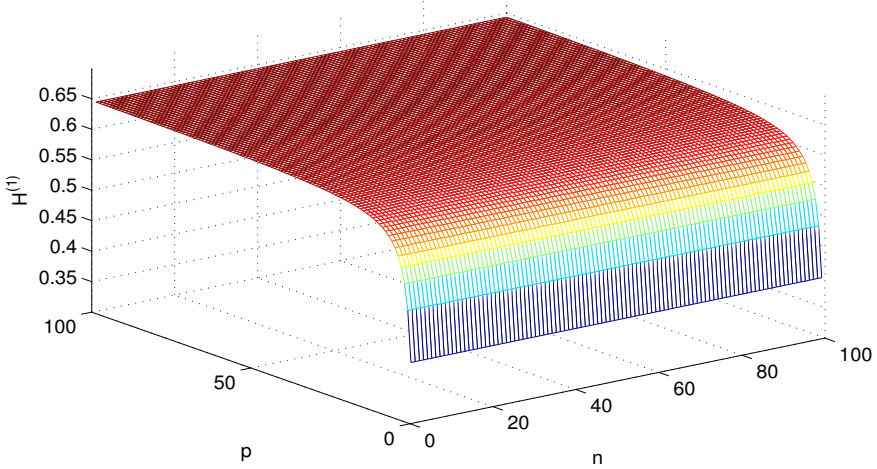

Figure 12. The change in $H^{(1)}$ for $\bar{B}$.

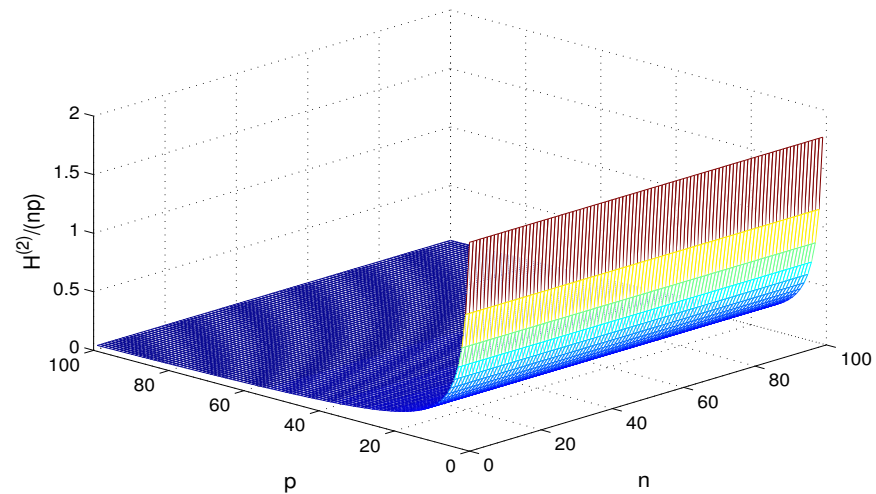

Figure 13. The change in $\frac{H^{(2)}}{p}$ for $\overline{\mathbb{B}}$. 


\section{Conclusions}

In this paper, the network coherence that characterizes the robustness of the triplex star-like works is studied through the methods of graph spectra; in addition, the algebraic connectivity that characterizes the convergence speed is discussed. By applying graph operations methods, two pairs of triplex star-like networks that have non-isomorphic graphs but with same structure parameters, i.e., $n$ and $k($ or $p)$, have been constructed. This paper mainly studies the asymptotic behavior of the network coherence of the triplex networks, and it is found that the adhering star topologies to each node of $\mathbb{A}$ and $\mathbb{B}$ will increase at a constant value to the first-order coherence. For the two groups of nonisomorphic graph structures, when $n \rightarrow \infty$, the first-order robustness of $\mathbb{B}$ is better than $\mathbb{A}$, and the second-order coherence of $\mathbb{A}$ and $\mathbb{B}$ is approximately equal as $n, k$ tends to infinity.

Author Contributions: D.H., H.J.; methodology, D.H., J.Z., Z.Y.; software, D.H., J.Z.; validation, D.H., Z.Y., H.J.; formal analysis, D.H., J.Z.; writing—original draft preparation, D.H., J.Z.; writingreview and editing, D.H., J.Z., Z.Y., H.J.; supervision, Z.Y., H.J.; project administration, D.H. All authors have read and agreed to the published version of the manuscript.

Funding: This work was supported by the Natural Science Foundation of Xinjiang (NSFXJ) (No.2019D 01B10), Youth Doctoral Cultivation Project for Sci-Tech Talents of Xinjiang (no.2018Q074).

Institutional Review Board Statement: Not applicable.

Informed Consent Statement: Not applicable.

Data Availability Statement: Some or all data, models, or code generated or used during the study are available from the corresponding author by request.

Acknowledgments: We express our sincere gratitude to the people who gave us valuable comments. This work was supported by the Natural Science Foundation of Xinjiang (NSFXJ) (No. 2019D01B10), Youth Doctoral Cultivation Project for Sci-Tech Talents of Xinjiang (No. 2018Q074).

Conflicts of Interest: The authors declare no conflict of interest.

\section{References}

1. Zhang, Y.; Tian, Y.-P. Consensus tracking in sensor networks with periodic sensing and switching connected topologies. Syst. Control Lett. 2015, 84, 44-51. [CrossRef]

2. Wen, G.; Chen, C.L.P.; Liu, Y.-J.; Liu, Z. Neural Network-Based Adaptive Leader-Following Consensus Control for a Class of Nonlinear Multiagent State-Delay Systems. IEEE Trans. Cybern. 2016, 47, 2151-2160. [CrossRef]

3. Liang, C.-D.; Wang, L.; Yao, X.-Y.; Liu, Z.-W.; Ge, M.-F. Multi-target tracking of networked heterogeneous collaborative robots in task space. Nonlinear Dyn. 2019, 97, 1159-1173. [CrossRef]

4. Saber, O.; Murray, R. Consensus problems in Networks of Agents With Switching Topology and Time-Delays. IEEE Trans. Autom. Control 2004, 49, 1520-1533. [CrossRef]

5. Ren, W. On consensus algorithms for double-integrator dynamics. IEEE Trans. Autom. Control 2008, 53, 1503-1509. [CrossRef]

6. Yu, W.; Chen, G.; Cao, M.; Kurths, J. Second-order consensus for multiagent systems with directed topologies and nonlinear dynamics. IEEE Trans. Syst. Man Cybern.-Part Cybern. 2010, 40, 881-891.

7. Wen, G.; Duan, Z.; Yu, W.; Chen, G. Consensus of second-order multi-agent systems with delayed nonlinear dynamics and intermittent communications. Int. J. Control 2013, 86, 322-331. [CrossRef]

8. Ma, J.; Zheng, Y.; Wang, L. LQR-based optimal topology of leader-following consensus. Int. J. Robust Nonlinear Control 2014, 25, 3404-3421. [CrossRef]

9. Li, J.; Feng, T.; Zhang, J.; Yan, F. Optimal distributed cooperative control for multi-agent systems with constrains on convergence speed and control input. Neurocomputing 2021, 426, 14-25. [CrossRef]

10. Sun, Y.; Li, W.; Zhao, D. Convergence time and speed of multi-agent systems in noisy environments. Chaos Interdiscip. J. Nonlinear Sci. 2012, 22, 43126. [CrossRef] [PubMed]

11. Young, G.F.; Scardovi, L.; Leonard, N.E. Robustness of noisy consensus dynamics with directed communication. In Proceedings of the 2010 American Control Conference, Baltimore, MD, USA, 30 June-2 July 2010; pp. 6312-6317.

12. Xiao, L.; Boyd, S.; Kim, S.-J. Distributed average consensus with least-mean-square deviation. J. Parallel Distrib. Comput. 2007, 67, 33-46. [CrossRef]

13. Patterson, S.; Bamieh, B. Network coherence in fractal graphs. In Proceedings of the IEEE Conference on Decision and Control and European Control Conference, Orlando, FL, USA, 12-15 December 2011; pp. 6445-6450.

14. Bamieh, B.; Jovanovi, M.R.; Mitra, P.; Patterson, S. Coherence in large-scale networks: Dimension-dependent limitations of local feedback. Trans. Autom. Control 2012, 57, 2235-2249. [CrossRef] 
15. Patterson, S.; Bamieh, B. Consensus and Coherence in Fractal Networks. IEEE Trans. Control Netw. Syst. 2014, 1, 338-348. [CrossRef]

16. Yi, Y.; Zhang, Z.; Shan, L.; Chen, G. Robustness of First- and Second-Order Consensus Algorithms for a Noisy Scale-Free Small-World Koch Network. IEEE Trans. Control Syst. Technol. 2017, 25, 342-350. [CrossRef]

17. Sun, W.; Ding, Q.; Zhang, J.; Chen, F. Coherence in a family of tree networks with an application of Laplacian spectrum. Chaos: Interdiscip. J. Nonlinear Sci. 2014, 24, 043112. [CrossRef]

18. Sun, W.; Li, Y.; Liu, S. Noisy consensus dynamics in windmill-type graphs. Chaos: Interdiscip. J. Nonlinear Sci. $2020,30,123131$. [CrossRef]

19. Dai, M.; Zhu, J.; Huang, F.; Li, Y.; Zhu, L.; Su, W. Coherence analysis for iterated line graphs of multi-subdivision graph. Fractals 2020, 28. [CrossRef]

20. Wang, X.; Xu, H.; Dai, M. First-order network coherence in 5-rose graphs. Physica 2019, 527, 121129. [CrossRef]

21. Zhou, B.; Liao, X. Leader-following second-order consensus in multi-agent systems with sampled data via pinning control. Nonlinear Dyn. 2014, 78, 555-569. [CrossRef]

22. Yu, Z.; Jiang, H.; Huang, D.; Hu, C. Consensus of nonlinear multi-agent systems with directed switching graphs: A directed spanning tree based error system approach. Nonlinear Anal. Hybrid Syst. 2018, 28, 123-140. [CrossRef]

23. Yu, Z.; Huang, D.; Jiang, H.; Hu, C. Consensus of second-order multi-agent systems with nonlinear dynamics via edge-based distributed adaptive protocols. J. Frankl. Inst. 2016, 353, 4821-4844. [CrossRef]

24. Hu, J.; Hong, Y.; Feng, G. Distributed dynamic control for leaderless multi-agent consensus with star-like topology. Asian J. Control 2008, 10, 233-237. [CrossRef]

25. Abdolyousefi, M.N.; Mesbahi, M. On the Controllability Properties of Circulant Networks. IEEE Trans. Autom. Control 2013, 58, 3179-3184. [CrossRef]

26. Wen, G.; Yu, W.; Wang, J.; Xu, D.; Cao, J. Distributed node-to-node consensus of multi-agent systems with time-varying pinning links. Neurocomputing 2015, 149, 1387-1395. [CrossRef]

27. Li, H.-L.; Cao, J.; Jiang, H.; Alsaedi, A. Graph theory-based finite-time synchronization of fractional-order complex dynamical networks. J. Frankl. Inst. 2018, 355, 5771-5789. [CrossRef]

28. Xu, M.; Lu, J.; Zhou, J. Synchronizability and eigenvalues of two-layer star networks. Acta Phys. Sin. 2016, 65, 028902.

29. Liu, H.; Xu, J.; Li, Z.; Wang, X.; Lu, J.; Zeng, Z. Optimizing Synchronizability of Multilayer Networks Based on the Graph Comparison Method. IEEE Trans. Circuits Syst. I Regul. Pap. 2020, 67, 1740-1751. [CrossRef]

30. He, W.; Xu, Z.; Du, W.; Chen, G.; Kubota, N.; Qian, F. Synchronization control in multiplex networks of nonlinear multi-agent systems. Chaos: Interdiscip. J. Nonlinear Sci. 2017, 27, 123104. [CrossRef]

31. Lu, R.; Yu, W.; Lu, J.; Xue, A. Synchronization on Complex Networks of Networks. IEEE Trans. Neural Netw. Learn. Syst. 2014, 25, 2110-2118. [CrossRef]

32. Hu, C.; Jiang, H. Cluster synchronization for directed community networks via pinning partial schemes. Chaos Solitons Fractals 2012, 45, 1368-1377. [CrossRef]

33. Hu, C.; He, H.; Jiang, H. Synchronization of complex-valued dynamic networks with intermittently adaptive coupling: A direct error method. Automatica 2020, 112, 108675. [CrossRef]

34. He, W.; Chen, G.; Han, Q.-L.; Du, W.; Cao, J.; Qian, F. Multiagent Systems on Multilayer Networks: Synchronization Analysis and Network Design. IEEE Trans. Syst. Man Cybern. Syst. 2017, 47, 1655-1667. [CrossRef]

35. Wang, Z.; Xia, C.; Chen, Z.; Chen, G. Epidemic Propagation With Positive and Negative Preventive Information in Multiplex Networks. IEEE Trans. Cybern. 2021, 51, 1454-1462. [CrossRef]

36. Kivelä, M.; Arenas, A.; Barthelemy, M.; Gleeson, J.P.; Moreno, Y.; Porter, M.A. Multilayer Networks. J. Complex Netw. 2014, 2, 203.

37. Barik, S.; Pati, S.; Sarma, B.K. The Spectrum of the Corona of Two Graphs. SIAM J. Discret. Math. 2007, 21, 47-56. [CrossRef]

38. Liu, Q. The Laplacian spectrum of corona of two graphs. Kragujev. J. Math. 2014, 38, 163-170. [CrossRef]

39. Douglas, B. West, Introduction to Graph Theory, 2nd ed.; Pearson Education: Hong Kong, China, 2004.

40. Huang, D.; Zhang, Z. On cyclic vertex-connectivity of Cartesian product digraphs. J. Comb. Optim. 2012, 24, 379-388. [CrossRef]

41. Cvetkovic, D.; Rowlinson, P.; Simic, S. An Introduction to the Theory of Graph Spectra; Cambridge University Press: Cambridge, UK, 2010.

42. Zhang, H.; Yang, Y. Resistance distance and Kirchhoff index in circulant graphs. Int. J. Quantum Chem. 2007, 107, 330-339. [CrossRef]

43. Xu, P.; Huang, Q. The Kirchhoff index of enhanced hypercubes. arXiv 2018, arXiv:1809.07189. 